\title{
The Morphology and Physics of the Local Interstellar Medium
}

\author{
FREDRICK C. BRUHWEILER
}

Department of Physics, The Catholic Univ, of America, Washington, DC 20064, USA

We are finally on the threshold of obtaining a coherent morphological and physical picture for the local interstellar medium (LISM), especially the region within $300 \mathrm{pc}$ of the Sun. The EUVE is playing a special role in revealing this picture. This instrument can provide direct measurements of the the radiation field that photoionizes both hydrogen and helium. It also can yield direct measurements of the column densities of hydrogen, but especially $\mathrm{He} \mathrm{I}$ and $\mathrm{He}$ II toward nearby white dwarfs. These observations suggest that the ionization in the Local Cloud, the cloud in which the Sun is embedded, is not in equilibrium, but in a recombination phase. Heuristic calculations imply that the the present ionization is due to the passage of shocks, at times greater than $3 \times 10^{6}$ years ago. The origin of these shocks are probably linked to the supernova which was responsible for the expanding nebular complex of clouds know as the Loop I supernova remnant, of which the Local Cloud is a part. extreme-UV radiation field, that which ionizes both hydrogen and helium in the LISM. Of the ISM within $300 \mathrm{pc}$, the volume appears to be predominantly filled by hot $\left(10^{6} \mathrm{~K}\right)$ coronal gas. This gas is laced with six largescale shell structures with diameters $\sim 100-150 \mathrm{pc}$ including the long-recognized radio loops, Loop I-IV, as well as the Orion-Eridanus and Gum Nebulae are identified. An idea that has evolved in the literature for over two decades is that the kinematically-linked OB associations representing Gould's Belt, plus the gas and dust of Lindblad's Ring, require that previous supernova activity and stellar winds carved out a 400-600 pc diameter cavity some 3 to $6 \times 10^{7}$ yr ago. This activity produced a pre-existing low density region, into which the present young loop structures have expanded. The outer boundaries of the identified expanding loop structures, inside this preexisting cavity, delineate the periphery of the the mis-named "local interstellar bubble." Thus, this picture naturally explains some of the problems often associated with the presence of this low density region exterior to Loop I.

\section{The Emerging Picture Within 50 pc}

The importance of the local interstellar medium is sometimes not fully appreciated by many astronomers, even though the study of this region is crucial to understanding the basic physical processes that arise in the interstellar gas at greater distances. For example, absorption studies for lines-of-sight toward nearby stars, where the distances are relatively certain, can we unambiguously determine the gas morphology and physical conditions in this gas. By studying long lines-of-sight, one usually does not know at what distance or in what cloud absorption lines arise. As we shall see, absorption studies with the $E U V E$ of $\mathrm{He}$ I and He II photoionization edges for objects within $50 \mathrm{pc}$ are now enabling us to place strong constraints on the basic ionization mechanisms for this gas.

Because stars within $50 \mathrm{pc}$ generally exhibit no detectable extinction and only negligible optical absorption, efforts to probe this region had to await for the advent of UV astronomy. Only then could the strong resonance lines of the cosmically abundant ionic species be seen. Consequently, practically all of our knowledge of the LISM within 50 pc has come from spacecraft.

The faint glow of the interstellar gas, immediately in the vicinity of the Sun (within $0.03 \mathrm{pc}$ ) can be seen through backscattering of radiation initially produced in the solar chromosphere by the strong emission lines of H I ( $\lambda 1216)$ and He I ( $\lambda 586)$. Analysis of this backscattered radiation indicates an interstellar hydrogen particle density near the 
Sun of $n(\mathrm{H} \mathrm{I}) \sim 0.1 \mathrm{~cm}^{-3}$, with a temperature of $T \approx 8-15,000 \mathrm{~K}$ (Bertaux et al. 1985; Chassefiere et al. 1988).

At greater distances, interstellar H I-L $\alpha$ absorption superposed upon the corresponding chromospheric emission of nearby late-type stars has provided a means to sample the gas within $\sim 5 \mathrm{pc}$ of the Sun. Besides yielding estimates for H I column densities, these studies have also obtained estimates for the cosmically important $\mathrm{D} / \mathrm{H}$ ratios for these lines-of-sight. Early investigations using the Copernicus satellite (McClintock et al. 1978, Dupree et al. 1978), and more recent studies using the IUE (Landsmann et al. 1985; Murthy et al. 1987) and HST (Linsky et al. 1993), are all consistent and yield $n(\mathrm{H} \mathrm{I})$ $\sim 0.1 \mathrm{~cm}^{-3}$ and $T \sim 10,000 \mathrm{~K}$. An analysis of the collected dataset also suggests that the density increases toward $l^{\text {II }} \sim 0^{\circ}$ in the general direction of Sco-Cen.

Within $\sim 50 \mathrm{pc}, I U E$ observations of nearby, hot white dwarfs (Bruhweiler \& Kondo 1982; also see Bruhweiler \& Kondo 1981 and Dupree \& Raymond 1982) have clearly indicated that neutral hydrogen is concentrated within a few parsecs of the Sun (the Local Cloud) with no other clouds detected within $50 \mathrm{pc}$. The largest detected H I column density for the white dwarfs studied was $N(\mathrm{H} \mathrm{I}) \approx 2 \times 10^{18} \mathrm{~cm}^{-2}$.

Almost every line-of-sight observed through the Local cloud shows multiple velocity components to interstellar lines as seen in high resolution spectral data acquired from the ground (Lallement et al. 1986, 1992) and from the Goddard High Resolution Spectrograph aboard the HST as any perusal of the archival data will show. Although this might be interpreted as evidence for multiple distinct clouds, given the implied history of this gas and the nature of the surrounding substate in which this gas is embedded, the multiple velocity components are more likely due to conduction fronts at the cloud interface (McKee \& Cowie 1977) and shocks recently passing through this cloud (also see Bruhweiler, Smith, and Lyu 1995).

Soft energy X-ray data reveal a uniform soft X-ray background with no evidence of cloud shadows (Fried et al. 1980; McCammon et al. 1983). The collected ultraviolet and soft X-ray data indicate the Local Cloud is surrounded by a hot, coronal gas at $T \approx 10^{6} \mathrm{~K}$ and $n \approx 10^{-2.5} \mathrm{~cm}^{-3}$.

Evidence for a conductive interface between the Local Cloud and the surrounding $10^{6} \mathrm{~K}$ gas is seen in GHRS data for the line-of-sight toward the B star, $\alpha$ Gru $(d=26$ pc). Weak interstellar features of C IV at levels of 3.5 and $1.7 \mathrm{~m} \AA$ are measured for the members of the resonance doublet, while a much stronger feature is seen in Si III (Bruhweiler, Smith, \& Lyu 1995). Since both of these ions have high charge-exchange rates with neutral hydrogen, they must arise in a pure $H$ II regions. The ratios of the implied column densities are compatible with simple conductive interface models.

A further comparison of the very low level optical polarization for nearby stars (Tinbergen 1981) and ultraviolet absorption line studies has revealed that the Sun is embedded in, near the edge of, a rather diffuse cloud, with a total extent less than $15 \mathrm{pc}$ (Bruhweiler 1982). For directions toward the hemisphere in the anti-galactic center direction, away from the main body of the Local Cloud, low $\mathrm{H}$ I column densities (i.e. $N(\mathbf{H ~ I})=1-2 \times 10^{18}$ $\mathrm{cm}^{-2}$ ) are intercepted through the outer cloud skin. Directions toward $l \approx 0^{\circ}$, intercepts the main body of the cloud and typically yield $N(\mathrm{H} \mathrm{I}) \approx 1-2 \times 10^{19} \mathrm{~cm}^{-2}$. The core of the Local Cloud lies near $l=5^{\circ}, b=-20^{\circ}$ (Also see Fig. 3 of Bruhweiler \& Vidal-Madjar 1987). The direction of the Local Cloud core also coincides with the region showing an absence of soft X-ray sources as seen in Rosat WPC data (Warwick et al. 1993). The absence of these sources is due to attenuation from gas corresponding to $N(\mathrm{H}$ I) $=1-2 \times 10^{19} \mathrm{~cm}^{-2}$.

Comparisons with radio data (discussed more thoroughly below) also show that the Local Cloud lies at the periphery and shares motion of the expanding filaments of the 
Loop I supernova remnant. This association with Loop I would make the Local Cloud a shell fragment, or perhaps better described as a wisp in the filaments of this supernova remnant.

\section{The Ionization of Hydrogen \& Helium in the Local Cloud}

Before the launch of the EUVE, Bruhweiler \& Cheng (1988) and Cheng \& Bruhweiler (1990) had calculated photoionization models to predict the ionization equilibrium of $\mathrm{H}$, He and heavy elements in the Local Cloud. Although we now have better estimates for the ionizing radiation fields, thanks for observations from the $E U V E$, these works are illustrative of what physical processes are important for the ionization of the two most abundant elements, $\mathrm{H}$ and $\mathrm{He}$.

In the calculations of Cheng \& Bruhweiler, contributions due to photoionization by the Extreme-UV/X-ray radiation field from the nearby hot stars and surrounding coronal substrate as well as collisional ionization, especially in the conductive interface of the Local Cloud skin are included. Effects of charge-exchange and Auger ionization are also considered. The high attenuation toward the hot stars of Sco-Cen precludes any ionizing flux shortward of the H I Lyman edge reaching the Sun from that direction. Thus, all the ionizing radiation is predicted to come from the direction of low column density, toward $l \sim 180^{\circ}$.

With the then "best guess" to the EUV radiation field, these calculations showed that the ionization fraction was higher for $\mathrm{He}$ than for $\mathrm{H}$ at the $\operatorname{Sun}\left(\mathrm{X}_{\mathrm{H}^{+}}=0.17\right.$ and $\mathrm{X}_{\mathrm{He}+}=0.3$ ). Specifically, it was found that the H-ionization was determined by the ambient stellar Extreme-UV radiation field, while the He-ionization was fixed by the diffuse radiation field from the surrounding hot coronal substrate. Specifically, it is the line emission in the hot substrate, principally from the Fe-complex near $190 \AA$ that was predicted to be the most significant source of ionization.

Recent Extreme-UV observations with HUT of the white dwarf, G191-B2B (Kimble et al. 1993), and both WD 2309+105 (Vennes et al. 1993) and GD659 (Holberg et al. $1995)$ are in striking agreement with these predictions. Yet, other results indicate that this agreement may be fortuitous.

$E U V E$ observations are now providing direct measurements for both the stellar and diffuse components to the EUV radiation field in the LISM. Perhaps the biggest surprise has been the discovery that the $\mathrm{B}$ star, $\epsilon \mathrm{CMa}$ is the dominant photoionizing source for hydrogen, much stronger than the numerous, hot, hydrogen-atmosphere DA white dwarfs. An estimate of the photoionization rate for $\mathrm{H}$ by summing the observed EUV fluxes from these stellar sources gives $\Gamma_{H I}=1.4 \times 10^{15} \mathrm{~s}^{-1}$ at the Sun (Vallerga \& Welsh 1995). this result is not too different from that predicted by Bruhweiler \& Cheng (1988). However, the contribution from the individual sources are definitely different than predicted, and the large contribution from $\epsilon \mathrm{CMa}$ was not foreseen.

The EUVE is also giving us some observational limits on the diffuse EUV radiation field capable of ionizing He in the LISM. The recent non-detection of Jelinsky et al. (1995) is a factor of 12 below the level predicted by Cheng \& Bruhweiler 1990). However, Jelinsky et al. did not recognize that the direction scanned in their observations overlap extensively with that of the Local Cloud core where high attenuation should be expected due to the $1-2 \times 10^{19} \mathrm{~cm}^{-2}$ column density for the main body of the cloud. For the emission line complex near $190 \AA$, this corresponds to an optical depth of $\tau_{190 \AA} \sim 1-2$. Notwithstanding, these observations still imply that the EUV flux is well below the Cheng \& Bruhweiler predictions. Conversations with J. Vallerga also indicate that other unpublished $E U V E$ observations of directions pointing away from the Local Cloud core 
show no diffuse EUV background radiation. Without further in depth analysis of these data, we can only estimate that the upper limit for the He ionization rate at the Sun is at least a factor of four below the Cheng \& Bruhweiler prediction. If so, this upper limit would make it highly unlikely that the observed He ionization can be due to ionization equilibrium.

If ionization equilibrium is ruled out, then one must assume that the observed $\mathrm{He}$ ionization reflects gas that is undergoing a time-dependent recombination. The initial high ionization would have been produced by a supernova explosion, presumably the one that gave rise to the observed Loop I remnant (also see Cox \& Anderson 1982). This ionizing event can be either a UV-flash or the passage of shocks through the gas now recognized as the Local Cloud.

We have recently performed time-dependent ionization calculations appropriate for the LISM (Lyu \& Bruhweiler 1995). in which we explored the ionization and recombination from both a photoionization pulse and shocks produced by a typical supernova in the Sco-Cen association.

Our calculations indicate that unless a supernova occurs at distances less than $20 \mathrm{pc}$ of a cloud, the cloud will not be significantly ionized. The high velocity mass loss from stars in Sco-Cen should have carved out a pre-existing hot cavity before the supernova explosion and the subsequent momentum of the ejecta drove the Sco-Cen shell to much larger radius. Conservative calculations indicate that the precursor cavity was much larger than $20 \mathrm{pc}$. Thus, the effects of any UV-flash should have been minimal.

The high velocity SN-ejecta after traversing the evacuated cavity would hit the surrounding clouds and compress them to high temperatures and high ionized levels. The peak temperatures in these high velocity shocks, as they pass through these clouds, should be well above $10^{5} \mathrm{~K}$. The temperature versus time profiles for gas at constant density above $10^{5} \mathrm{~K}$ are very similar for a wide range of conditions (cf., Shapiro \& Moore 1977). Our models for the recombining $\mathrm{H}$ and He below $10^{5} \mathrm{~K}$, with the effects of the EUV radiation field included, can easily reproduce the deduced $\mathrm{H}$ and $\mathrm{He}$ ionization fractions deduced from $E U V E$ observations of sightlines toward hot white dwarfs (see above). For both the observed ionization fractions and the limits for the ionizing fluxes, acceptable fits are obtained for 2 to 3 million years since the beginning of the recombination phase (defined to be the time when $T$ dropped below $10^{5} \mathrm{~K}$ ). Other assumptions place the time when the supernova event occurred at roughly 3.8 million years ago. These calculations indicate that time-dependent ionizations can give a reasonable explanation to the observed $\mathrm{H}$ and $\mathrm{He}$ ionization fractions deduced from $E U V E$ data.

The EUVE is basically a Helium machine. The wavelength coverage of its spectrographs spans both the ionization edges of $\mathrm{He}$ I and He II. For hot white dwarfs with significant fluxes down to $228 \AA$, there is the possibility of measuring both the absorption of He I and He II toward the same object. For objects with large H I column densities such that the He I edge at $504 \AA$ experiences large IS attenuation, the He I autoionization feature at $204 \AA$ can be used (cf., Vennes et al. 1993; Holberg et al. 1995).

All the information about the He ionization fractions are derived from observations of hot hydrogen atmosphere (DA) white dwarfs. However, one major complication in using these objects is the observed opacity from trace metals below $300 \AA$. This opacity is mainly due to photospheric absorption from resonance lines and low-lying metastable levels of $\mathrm{C}, \mathrm{N}, \mathrm{O}$, and Fe-peak elements. However many of the contributors have not been identified. Until proper line-blanketed non-LTE models becomes available, it will be difficult to obtain reliable He ionization ratios for the interstellar gas toward these objects. Thus, the current reliable estimates have come from white dwarfs which show 
little hint of metals in their spectra; such objects as GD659. (Also see contributions by I. Hubeny and M. Barstow, these proceedings.)

\section{The Local Supershell Structures \& the Origin of the Local Bubble}

\subsection{Determining Gas Morphology Out to $300 \mathrm{pc}$}

Delineating the gas morphology beyond $50 \mathrm{pc}$ requires a synthesis of observations spanning the electromagnetic spectrum. Perhaps the best clues to this structure of the LISM comes from radio wavelengths. The radio loops, Loop I through Loop IV, are all contained within 250-300 pc (Berkhuijsen 1971; Spoelstra 1972). The most notable of the radio loops is Loop $\mathrm{I}$, which spans more than $100^{\circ}$ of the sky. The interior of Loop $\mathrm{I}$ is the most prominent diffuse soft $\mathrm{X}$-ray source $\left(\approx 90^{\circ}\right.$ in diameter $)$ seen in the $\mathrm{M}$ band $(440-930 \mathrm{eV})$ of the full-sky Wisconsin sounding rocket data (McCammon et al. 1983). These combined results demonstrate Loop I is filled with hot gas, hotter than that seen in all directions at lower X-ray energies. Loop I is thought to have been produced by a recent supernova that occurred in the Sco-Cen stellar association within the last few $10^{6} \mathrm{yr}$. As previously mentioned, the position of the Local Cloud and its implied motion suggest that it is a shell fragment of Loop I. We emphasize that the low measured column densities, $N(\mathrm{H} \mathrm{I}) \leq 2 \times 10^{18} \mathrm{~cm}^{-2}$, are measured in the direction away from Loop I in the region often labeled the "Local Bubble" by many. This suggests that the shell complex of Loop $\mathrm{I}$ is actually expanding into a hot $\left(10^{6} \mathrm{~K}\right)$, pre-existing low density region.

Just where the observed diffuse $\mathrm{X}$-ray emission originates is not necessarily straightforward. However, IRAS, Radio, UV absorption line, and extinction results all point to significant concentrations of cool gas at distances beyond $100 \mathrm{pc}$ in the direction of the Sco-Cen, especially near $l \sim 30^{\circ}$ and the clouds where the stars $\zeta$ Oph and $\rho$ Oph are found (see de Geus 1991). Since the emission measure is proportional to $\left(n_{e}\right)^{2}$, the observed $\mathbf{X}$-ray emission in the $\mathbf{M}$ band probably arises in front of, on or near, the dense clouds close to the back wall of the Loop I cavity.

The shells representing Loop I and the other loops are not like the surfaces of a balloon, but are more like a complex of expanding cloud fragments. Thus, the hotter gas of Loop I's interior is probably freely mixing with the hot gas exterior to Loop I.

Determining temperatures of the $\mathrm{X}$-ray emitting gas is not a simple matter. Most of the X-ray flux at temperatures of interest here $\left(10^{6} \leq T \leq 10^{7} \mathrm{~K}\right)$ is emitted as line emission. But the ion recombination times for this low density plasma are quite long. Thus, the ionic abundances, as for $\mathrm{H}$ and $\mathrm{He}$, are not represented by electron temperature, but depend upon the ionization history of the gas. The actual emission line intensities should be far different from those predicted from ionization equilibrium. Surely as the spectral resolution improves in new X-ray instrumentation, much that we have inferred about the characteristics of this gas may well change (see Edgar et al. 1993 and also this conference).

Besides the four radio loops, Loop I-IV, two additional shell structures are also found within $300 \mathrm{pc}$. The stellar winds and supernovae from stars in the Orion OB1 association ( $d \sim 400 \mathrm{pc}$ ) have produced a large expanding shell complex (Cowie et al. 1979) spanning the Orion and Eridanus constellations. This complex is defined by Barnard's Loop on the East and a system of nebular filaments traced out in [O III] and $\mathrm{H}-\alpha$ extending to the West in the sky (Reynolds \& Ogden 1979). Burrows et al. (1993) in a re-interpretation of these data, incorporating $\mathrm{X}$-ray and IRAS observations, suggest that this complex may extend to within $100 \mathrm{pc}$ (near $l \sim 180^{\circ}$ ) of the Sun.

Another nearby shell complex is the well-known Gum Nebula (Brandt et al. 1977; 


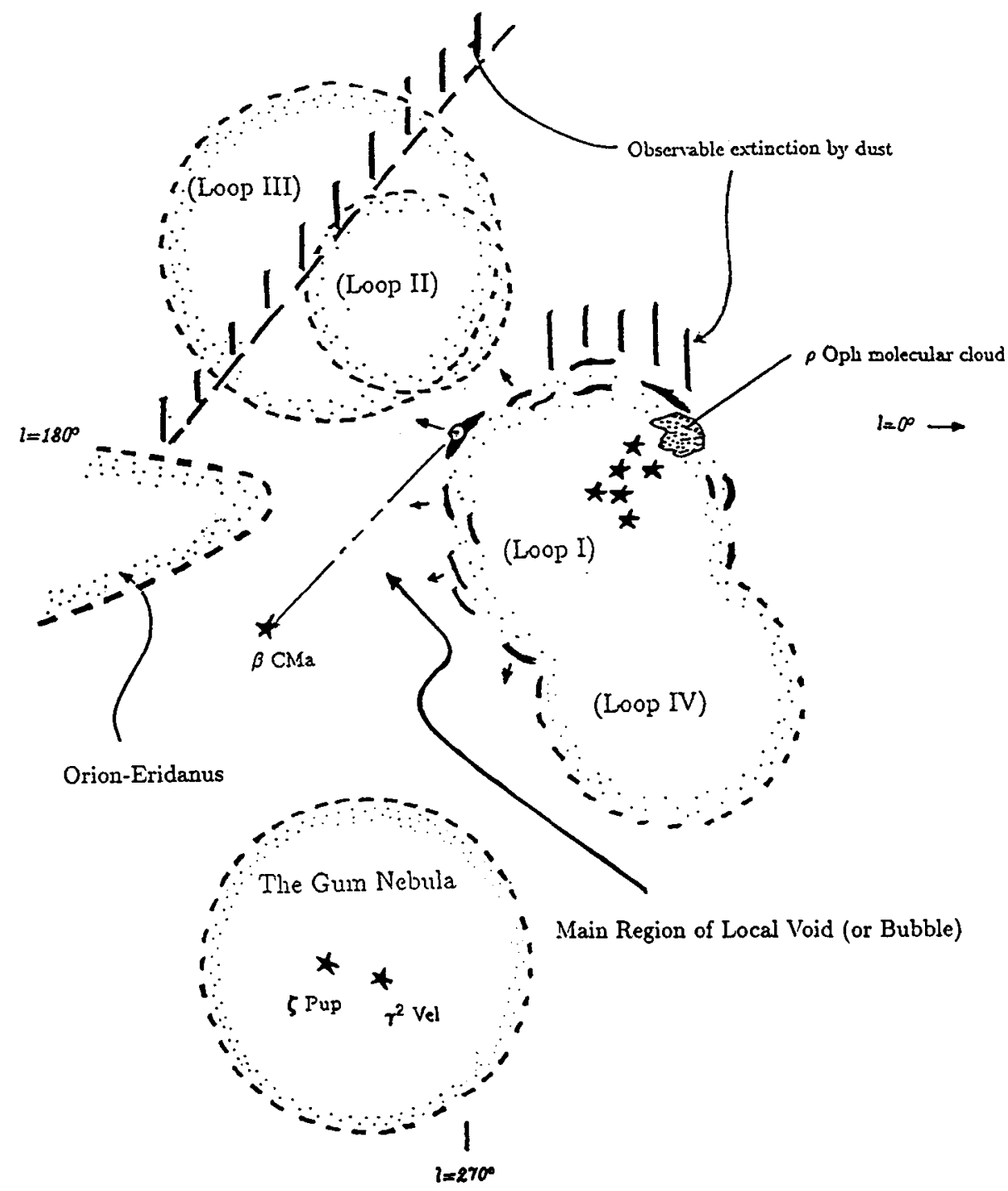

FIGURE 1. Distribution of largescale shell structures in the LISM. The sizes and placement of the radio loops, Loops I-IV were determined from Spoeltra (1973). A portion of the Orion-Eridanus Complex, as well as the Gum Nebula, are shown. The line-of-sight between the Sun and $\beta \mathrm{CMa}$ is depicted. This denotes the principal region of the Local Void (or Bubble). See text for discussion.

Reynolds 1977). This structure, seen near $l=270^{\circ}$, is centered about $400 \mathrm{pc}$ away and has a radius of $120 \mathrm{pc}$ as defined by the $\mathrm{H}-\alpha$ filaments. This nebula and its cavity contain the stars $\zeta$ Pup (O4) and $\gamma^{2} \mathrm{Vel}(\mathrm{WR}+\mathrm{O} 9 \mathrm{Ia})$. The stellar winds from these stars appear to be responsible for carving out the observed cavity (cf., Weaver et al. 1977). The Vela supernova remnant lies either in, or more likely behind, the Gum Nebula.

It appears that Loop $I$ is expanding into a pre-existing hot, coronal region, which 
extends at least $200 \mathrm{pc}$ in the direction of $\beta$ and $\epsilon \mathrm{CMa}$. (This is the region often referred to as the Local Bubble.) As illustrated in Figure 2, the term, Local Bubble, is clearly a misnomer, for the its boundary actually seems to denote the peripheries of the expanding neighboring shell complexes. Thus, the Local Bubble, itself, is not a bubble.

\subsection{What Is the Origin of the Pre-Existing Cavity?}

If the Local Bubble (or Void) is part of a pre-existing low density region filled with $10^{6} \mathrm{~K}$ gas, then what produced this region? One possibility is that the entire region within $400-600 \mathrm{pc}$ of the Sun was previously the site of a much older, much more energetic shell complex. This shell may have triggered the star formation now represented by the kinematically expanding Gould's Belt. Gould's Belt is defined by the stellar associations of Orion, Perseus, Lacertae, and Sco-Cen, some of which lie outside the plane (Blaauw 1956; Hughes \& Routledge 1972; Lesh 1972). Also, an expanding ring of gas and dust, called Lindblad's Ring has been linked with these stellar associations (Lindblad 1973; FitzGerald 1968; Olano 1982). More recently, Taylor, Dickman, Scoville (1987) has found that the molecular clouds, too, show this same kinematic expansion.

If it does, the inferred age of this supershell would be in 3 to $6 \times 10^{7} \mathrm{yr}$ (Elmegreen 1982). The original stellar associations responsible for carving out this large shell complex may have been part of the Taurus group and the $\alpha$ Per cluster. Unfortunately, most of the "smoking guns," namely the stars giving rise to the stellar winds and supernovae driving the expansion of this yet putative shell, are long gone.

Both Gould's Belt and Lindblad's Ring have been topics in the literature for years, and may present evidence that the region now within $400-600$ pc was previously evacuated and filled with hot coronal gas. An old pre-evacuated cavity would allow any shells, carved out by the stellar winds and supernovae from the young nearby stellar associations, a natural setting for expansion.

\section{Summary}

Multi-frequency observations, radio through X-ray wavelengths, have been essential in revealing the gas and dust morphology within $300 \mathrm{pc}$. Observations with the EUVE are revealing much about the ionization and physical conditions of the two most fundamental elements, $\mathrm{H}$ and $\mathrm{He}$, in the LISM. Within $300-450 \mathrm{pc}$, there are six identified large shell nebulae, which appear to be expanding into a pre-existing hot, low density cavity. This older cavity may owe its origin to stars of the $\alpha$ Per cluster, members of the Taurus moving group. This scenario provides a natural explanation for the misnamed "Local Bubble," which has been inferred to explain the soft X-ray background and the low H I column densities seen in the LISM in the galactic anti-center direction.

Thanks are extended to my fellow colleagues, K.-P. Cheng, J. Holberg, C.-H. Lyu, Y. Kondo, \& A. Smith, who I have had the pleasure of working with is aspects of this work.

\section{REFERENCES}

BERKHUiJSEN, E. 1971, A\&A, 14, 359

Bertaux, J. ET AL. 1985, A\&A, 150, 82

BlaAuw, A. 1956, ApJ, 123, 406

Borlun, R. ET AL. 1976, ApJ, 224, 132

BRANDT, J. ET AL. 1977, ApJ, 208, 109 
BruHweller, F. 1982, in Advances in Ultraviolet Astronomy, Based on Six Years of IUE Research, ed. Y. Kondo, R. Chapman, \& J. Mead, NASA, 125

Bruhweiler, F., \& Cheng, K. -P. 1988, ApJ, 335, 188

BRUHWEILER, F., \& KoNDO, Y. 1981, ApJL, 248, L123

Bruhwetler, F., \& Kondo, Y. 1982, ApJ, 259, 232

Burrows, D. 1993, ApJ, 406, 97

Cheng, K. -P., \& BruhweILER, F. 1990, ApJ, 364, 573

Cox, D., \& ANDErson, P. 1982, ApJ, 252, 268

Chassefiere, E. et al. 1988, A\&A, 174, 239

DE Geus, E. 1988, Ph.D. Dissertation

Dupree, A., \& RaYmond, J. 1982, ApJL, 263, L63

EDGAR, R. J. ET AL. 1993, BAAS, 25, 805

Elmegreen, B. 1982, in Submillimetre Astronomy, ed. J. Beckman,\& J. Philips, Cambridge Press, 3

Fried, P. M. ET AL. 1980, ApJ, 242, 987

Gry, C. ET AL. 1985, ApJ, 296, 593

Hughes, V., \& Routledge, D. 1972, AJ, 77, 210

KmMBLe, R. ET AL. 1993, ApJ, 404, 663

Lallement, R., Bertaux, J. L., Sandel, B. R., \& Chassefiere, E. 1992, in Solar Wind Seven, Proc. of the 3rd COSPAR Colloq., Goslar, Germany, (A93-33554 13-92), 209

LALLEMENT, R. ET AL. 1986, A\&A, 168, 225

LESH, J. 1972, ApJS, 17, 371

LINDBLAD, P. 1973, A\&A, 24, 309

LINSKY, J. ET AL. 1993, ApJ, 402, 694

MCCAMmon, D. eT AL. 1983, ApJ, 269, 107

Murthy, J. ET AL. 1987, ApJ, 315, 675

Olano, R. 1982, A\&A, 112, 195

Perry, C. et AL. 1982, AJ, 87, 1751

REYNolds, R. 1976, ApJ, 203, 151

ReYnolds, R. 1989, ApJL, 339, L29

Reynolds, R., \& Ogden, J. 1979, ApJ, 229, 942

Spoelstra, J. 1973, A\&A, 24, 149

TAYLOR, J. ET AL. 1987, ApJ, 324, 149

Tinbergen, J. 1982, A\&A, 105, 53

VenNes, ET AL. 1993, ApJ, 400,400

WARWICK, ET AL. 1993, MNRAS, 100, 100

Weaver, R. et al. 1977, ApJ, 218, 377 